\title{
Philosophiques
}

M. Weber, La Domination, traduit de l'allemand par Isabelle Kalinowski, note éditoriale d'Yves Sintomer, Paris, Éditions La Découverte, coll. "Politiques \& Sociétés ", 2013, 427 pages

\section{Marc-Kevin Daoust}

Volume 41, numéro 1, printemps 2014

URI : https://id.erudit.org/iderudit/1025735ar

DOI : https://doi.org/10.7202/1025735ar

Aller au sommaire du numéro

Éditeur(s)

Société de philosophie du Québec

ISSN

0316-2923 (imprimé)

1492-1391 (numérique)

Découvrir la revue

Citer ce compte rendu

Daoust, M.-K. (2014). Compte rendu de [M. Weber, La Domination, traduit de l'allemand par Isabelle Kalinowski, note éditoriale d'Yves Sintomer, Paris,

Éditions La Découverte, coll. « Politiques \& Sociétés », 2013, 427 pages].

Philosophiques, 41(1), 223-228. https://doi.org/10.7202/1025735ar d'utilisation que vous pouvez consulter en ligne.

https://apropos.erudit.org/fr/usagers/politique-dutilisation/ 
progressive du monde objectif par l'esprit fini. Par rapport à ce projet, le livre de Tinland fait office, comme il le reconnaît lui-même, de prolégomènes. Il ne produit pas l'odyssée idéaliste de l'esprit, le système hégélien en sa concrétude, mais une clarification des concepts structurant le système. À notre sens, les thèses de l'auteur gagneraient à s'appuyer sur une analyse plus fournie du langage chez Hegel. Tout le livre semble dominé par l'idée que Hegel aurait produit un discours au fait de ses conditions discursives. Or nulle part n'est vraiment analysée la fonction que joue le langage chez Hegel, et l'usage qu'il en fait. Le philosophe allemand, dans la préface à la seconde édition de sa Science de la logique, ne manquait pourtant pas de donner des indications quant à ce problème. Par ailleurs, une littérature non négligeable a été consacrée à ce sujet ${ }^{3}$, ce que l'auteur néglige en ne citant que le livre de Lebrun. On peut également regretter que certains concepts propres à la logique hégélienne, comme ceux de la relation ou de la vie, soient peu thématisés. Mais il est évident que l'on ne peut prétendre à l'exhaustivité quand on traite d'un thème aussi central que ne l'est l'idéalisme pour une philosophie dont l'ampleur et la complexité sont largement reconnues.

Hormis ces remarques, somme toute assez accessoires, touchant à la complétude de cette introduction à l'idéalisme hégélien, on ne peut donc que saluer la clarification qu'apporte l'auteur quant à des thèmes majeurs de la philosophie hégélienne et de sa réception. Il s'agit donc d'un ouvrage particulièrement bienvenu qui préviendra de nombreux malentendus dans l'interprétation et qui situera les principaux enjeux de la pensée du philosophe allemand. Nul doute qu'il ne devienne à terme un classique de la littérature hégélienne.

GUILLAUME LEJEUNE

Université Libre de Bruxelles

M. Weber, La Domination, traduit de l'allemand par Isabelle Kalinowski, note éditoriale d'Yves Sintomer, Paris, Éditions La Découverte, coll. «Politiques \& Sociétés », 2013, 427 pages.

Les manuscrits originaux à partir desquels est publié l'ouvrage La domination datent du début des années I9I0. Ces manuscrits devaient être une composante de la vaste entreprise gravitant autour d'Économie et société. Weber est toutefois emporté par la maladie avant la publication de ces

3. Pour une bibliographie détaillée sur le langage chez Hegel, on consultera: Guillaume Lejeune, Sens et usage du langage chez Hegel, Paris, Hermann, 20 I 4. 
œuvres. La domination ne sera achevée qu'après la mort de Weber, par sa femme Marianne Weber ${ }^{1}$.

Dans les années I990, de nombreux fragments de l'œuvre sont disponibles en langue française. Toutefois, aucune édition ne propose une traduction rigoureuse de l'ensemble des textes. Ce n'est qu'en $20{ }_{3} 3$ qu'est complétée pour la première fois l'actuelle traduction française complète de La domination. Remercions Isabelle Kalinowski pour sa traduction rigoureuse et claire de cette œuvre attendue. Son travail entourant les manuscrits est d'une grande pertinence. Par exemple, à plusieurs reprises, Kalinowski clarifie les périodes historiques et les principaux acteurs des événements mentionnés. La traductrice précise aussi si les sections sont traduites à partir de manuscrits achevés, ou si, au contraire, il s'agit de sections incomplètes. Ces commentaires fouillés sont d'une très grande utilité pour circuler rapidement et aisément dans l'œuvre.

Cette traduction est précédée d'une introduction critique d'Yves Sintomer, qui souligne à juste titre que La domination ne remettra pas notre compréhension de Weber en cause. Par contre, l'ouvrage introduit des définitions structurées, et son approche ressemble davantage à un système, à un ensemble d'idées logiquement solidaires. Cette lecture contribue donc à enrichir notre lecture des concepts et approches classiques de la sociologie wébérienne.

L'analyse en cours vise à dégager les idées essentielles de Weber dans La domination, pour ensuite montrer la pertinence philosophique d'une telle lecture.

Weber définit la domination comme une relation sociale, un rapport entre plusieurs personnes. Pour qu'il y ait domination, il faut qu'au moins un individu commande une classe d'individus dominés qui doivent, le cas échéant, obéir ${ }^{2}$. Ce qui intéresse surtout Weber, c'est une forme particulière de domination, soit la domination par l'autorité. D'un point de vue structurel, la domination apparaît lorsqu'une classe dominée accepte et intériorise le rapport d'autorité ${ }^{3}$. Avec raison, Sintomer nous indique qu'il serait vain de chercher à comprendre les mouvements contestataires de masse à partir des catégories proposées par Weber ${ }^{4}$. L'obéissance, en particulier l'élitisme, est un aspect central de La domination.

Les lieux où s'exprime la domination sont divers. On peut penser, en outre, à la famille, aux lieux de culte, aux lieux d'enseignement, à la fonction publique ou à l'entreprise privée 5 . On peut tout de même dégager une synthèse de ces réalités diverses, en catégorisant les types de domination

1. M. Weber, La domination, $20 \mathrm{I}_{3}$, p. 5 .

2. M. Weber, La domination, 20I3, p. 44-45, 49.

3. Ibid., p. 46, 49.

4. Ibid., p. 27.

5. Ibid., p. 43, 45 . 
selon des schèmes idéaux. Les catégories nous permettent alors d'analyser des "réalités multiples ${ }^{6}$ » à l'aune de critères communs. Il serait toutefois surprenant que la réalité colle parfaitement à ces constructions théoriques. Ainsi, les faits observables seront une approximation pertinente des catégories idéalisées ${ }^{7}$.

La domination bureaucratique est un mode de domination stable et caractérisé par le recours à des objectifs rationnels et impersonnels. Les fonctionnaires de ce système sont recrutés à titre de spécialistes et ont un champ d'action défini par cette expertise ${ }^{8}$. Ce type de domination est stable et rationnel, notamment puisqu'il se fonde sur une hiérarchie stricte, qu'il propose une gestion des tâches et des actions par écrit, et qu'il emploie un corps de fonctionnaires spécialisés ${ }^{9}$ dont la loyauté s'exprime à l'égard d'une "finalité objective impersonnelle ${ }^{10}$ ». Ce mode de domination tend à s'imposer dans le temps de par sa capacité à minimiser les coûts, sa recherche de précision, d'archivage et de collecte, sans oublier son caractère permanent et unifié. Weber, dans un possible souci de neutralité, note qu'il s'agit d'une des "structures sociales les plus difficiles à détruire ${ }^{11}$ ", et ce, exactement pour les mêmes raisons qu'elle s'impose dans le temps ${ }^{12}$.

La domination patrimoniale est une structure pré-bureaucratique. Comme la domination bureaucratique, ce mode de domination est relativement stable dans le temps. Ce qui la distingue radicalement est qu'elle est fondée sur la tradition, soit "la croyance dans le caractère intangible de ce qui a toujours été ainsi ${ }^{13}{ }^{\prime}$. Le sujet se soumet au souverain, et le souverain revendique ce droit dans l'existence de normes traditionnelles, rendues sacrées par des autorités morales. L'administration patrimoniale gravite autour des "besoins matériels purement personnels et essentiellement privés du maître ${ }^{14}$ ». La séparation entre sphère privée et sphère professionnelle est généralement inexistante ${ }^{15}$. Il s'agit essentiellement d'un mode de domination en rapport au souverain et sa cour. Pour cette raison, plus le territoire du souverain est grand, plus les régions éloignées du siège du pouvoir sont indépendantes ${ }^{16}$.

Les rapports de soumission peuvent s'essouffler avec le temps. L'obtention d'une rémunération indépendante de la volonté personnelle du souve-

6. Ibid., p. 38 .

7. Ibid., p. 38-39.

8. Ibid., p. 63 .

9. Ibid., p. 64-66.

10. Ibid., p. 66.

11. M. Weber, La domination, 2013, p. Iо०.

12. Ibid., p. IO०-IO3.

13. Ibid., p. I2 I.

14. Ibid., p. I 3 I.

15. Ibid., p. I 52.

16. Ibid., p. I8I. 
rain tend, entre autres, à affaiblir l'influence du souverain sur son administration ${ }^{17}$. Il se peut aussi que la tradition s'impose au souverain, ce qui introduit une "stéréotypisation et une stabilisation ${ }^{18}$ " des relations sociales entre les groupes sociaux. Les demandes arbitraires du souverain en tant qu'individu disparaissent et sont remplacées par un ensemble de règles stables et permanentes. La permanence des règles est rendue possible par la maîtrise de la diffusion écrite et calculatoire des règles ${ }^{19}$. Weber qualifie alors le mode de domination féodal comme un «cas limite» du régime patrimonial, une étape de transition entre le régime patrimonial et la domination bureaucratique ${ }^{20}$.

Si la domination bureaucratique se caractérise par la stabilité et la rationalité, et la domination patrimoniale par la stabilité et l'absence de rationalité, on peut imaginer une autre permutation, soit une domination irrationnelle et instable. C'est le cas de la domination charismatique. Dans une situation de "détresse psychique, physique, économique ${ }^{21}$ » apparaissent des "porteurs de dons spécifiques du corps et de l'esprit ${ }^{22}$ ", dons qui ne sont pas accessibles à tous. Weber précise toutefois que la domination charismatique ne peut prendre place si les dominés n'ont pas la conviction émotionnelle qu'il existe une entité supérieure à eux, bref, s'ils n'ont pas une confiance sans réserve en une figure héroïque ${ }^{23}$. Le leader charismatique construit une structure sociale et administrative autour de ses succès et, de la même manière, est responsable personnellement des échecs de son administration ${ }^{24}$. Ce régime est bref ou instable, et tend à disparaître plus facilement que les précédents modes de domination ${ }^{25}$. L'étude des différents mécanismes de préservation de la domination charismatique est toutefois remarquable. On peut penser à l'étude du charisme de fonction ou à la préservation du charisme par la discipline ${ }^{26}$.

Bien que l'œuvre se présente sous un angle éminemment sociologique, elle présente un intérêt philosophique manifeste. Cet ouvrage présente l'épistémologie critique, mais aussi l'approche idéal-typique et compréhensive de Weber. Au moment où Weber rédige ces manuscrits, l'école historique allemande développe et défend vigoureusement des sciences sociales empiriques "démarquées de la philosophie ${ }^{27}$ ", alors que le marxisme connaît un essor

17. Ibid., p. 156.

18. Ibid., p. 209.

19. Ibid., p. 236.

20. M. Weber, La domination, 2013, p. 209.

21. Ibid., p. 270.

22. Ibid.

23. Ibid., p. 279.

24. Ibid., p. 274.

25. Ibid., p. 289.

26. Ibid., p. 320, 338-34I.

27. Ibid., p. I3. 
hors du milieu universitaire. Dans ce contexte, l'approche sociologique retenue par Weber n'est pas consensuelle. La domination est alors l'occasion pour Weber de clarifier et de justifier sa méthode.

La rédaction des manuscrits composant La domination s'effectue à un stade "pré-paradigmatique» des sciences sociales, pour employer un terme étranger à Weber. L'auteur y défend donc sa méthode, ainsi qu'une conception particulière de la sociologie. À titre d'exemple, La domination illustre aussi à merveille le travail sur les catégories en sociologie, une méthode encore très actuelle. Les catégories y sont des artifices permettant un découpage de la réalité selon des caractéristiques données. Le rapport aux études empiriques ne se limite toutefois pas à "classifier» la réalité. À plusieurs reprises, Weber compare les développements historiques de scénarios similaires pour distinguer les aspects structurels des aspects singuliers d'une catégorie. C'est le cas, notamment, lorsque Weber compare le fonctionnement des administrations patrimoniales en Égypte ancienne, dans l'empire chinois, dans la Russie tsariste, ainsi que dans l'Angleterre du XII ${ }^{e}$ siècle ${ }^{28}$. Si Weber pense que le souverain, dans la domination patrimoniale, cherche à préserver sa domination sur les notables et les personnes privées, il constate que les stratégies, les conflits et les rapports d'influence pour y arriver sont très différents d'une étude de cas à l'autre. Dans La domination, Weber clarifie en quelque sorte le rapport entre construction scientifique a priori et analyse empirique. L'intérêt philosophique d'une lecture comme La domination est donc d'exposer et de comprendre les raisons scientifiques derrière la sociologie wébérienne. Si des ouvrages comme les Essais sur la théorie de la science ou Le savant et le politique ont théorisé la méthode et l'épistémologie des sciences sociales, La domination met ces demandes en pratique et les justifie sur une base immédiate.

Comme l'indique Sintomer, Weber ne propose pas une épistémologie spécifique à l'économie ou à la sociologie, mais propose plutôt de l'intégrer "comme une composante des sciences sociales, sur la base d'une approche conceptuelle et méthodologique globale ${ }^{29}$ ». Sa démarche se veut donc générale, et applicable à une classe entière de sciences, à savoir les sciences dont l'objet d'étude est la réalité humaine. L'effort de généralité se remarque aussi dans une tentative d'échapper à l'eurocentrisme. Pour construire les types idéaux de la domination, Weber se réfère non seulement à des États et des structures politiques européennes, mais cite en exemple des pays sur tous les continents. On peut toutefois se demander si l'eurocentrisme est réellement évité. Weber présente la domination bureaucratique comme l'évolution normale, sinon le stade achevé des autres formes de domination. Au même moment, le capitalisme se déploie partout en Occident. Difficile de croire que, par hasard, c'est le stade de développement auquel arrive la société

28. M. Weber, La domination, 2013, p. I72.

29. Ibid., p. I9. 
occidentale au début du $\mathrm{xx}^{\mathrm{e}}$ siècle. À cet égard, une des grandes forces du commentaire de Sintomer consiste par ailleurs à montrer le biais androcentriste, eurocentriste, voire ethnocentriste de La domination ${ }^{30}$. L'auteur précise toutefois, à juste titre, que l'on observe ces biais dans l'œuvre de Weber grâce au "privilège d'un siècle de recul ${ }^{31}$ ". Ajoutons aux remarques de Sintomer que nous exposons et rejetons ces biais précisément parce que nous croyons qu'ils privent la science de la généralité qui lui est propre. En ce sens, même si nous constatons que la thématisation politique chez Weber est dépassée, notre attitude à l'égard de son œuvre ne saurait être plus wébérienne.

La recherche de généralité suppose enfin une démarche neutre, ce qui explique pourquoi Weber propose une lecture des modes de domination dénuée de jugements de valeur. Même s'il est régulièrement affirmé, l'objectif de neutralité n'est pas toujours évident. À plusieurs reprises, Weber emploie des concepts éthiques denses. Par concept dense, on fait référence à des concepts qui ont une dimension descriptive, mais aussi une connotation normative. On peut penser, notamment, à des concepts comme l'irrationalité, la puissance, la suprématie, ou la force créatrice. Weber précise toutefois qu'il emploie ces concepts dans une perspective empirique stricto sensu. Il est aujourd'hui controversé d'affirmer que l'on peut employer des concepts éthiques denses sans tenir compte de leur dimension normative. On comprend toutefois que Weber cherche à comprendre et à rationaliser l'état d'esprit dans lequel se trouve le dominé pour justifier sa soumission à un ordre donné. En d'autres termes, la démarche de Weber fait appel aux valeurs subjectives de l'agent, et vise à comprendre le sens de ces valeurs dans un rapport à l'autorité. Il s'agit d'une piste pertinente pour parer à la critique épistémologique de la neutralité axiologique.

Cette contribution rigoureuse de Sintomer et Kalinowski est un incontournable de la méthodologie et de la sociologie wébérienne en langue française.

MARC-KEVIN DAOUST

Université de Montréal marc-kevin.daoust@umontreal.ca

30. M. Weber, La domination, 20I3, p. 34.

31. Ibid., p. 37. 